\title{
ESTUDO DA ESTRUTURA INTERNA E PRECISÃO DA ESCALA BALBINOTTI-CARDOSO DE SUPORTE SOCIAL DE TREINADORES (EB-CSST- 08)
}

\author{
Marcelo Silva Cardoso \\ Universidade Federal do Rio Grande do Sul, Porto alegre, Rio Grande do Sul, Brasil. \\ Marcos Alencar Abaide Balbinotti \\ Université du Québec à Trois-Rivières, Trois-Rivières, Québec, Canadá. \\ Daniela Wiethaeuper \\ Université du Québec à Trois-Rivières, Trois-Rivières, Québec, Canadá. \\ Carlos Adelar Abaide Balbinotti \\ Universidade Federal do Rio Grande do Sul, Porto alegre, Rio Grande do Sul, Brasil.
}

\begin{abstract}
Resumo
O objetivo deste estudo é testar o modelo bidimensional - Orientação à Confiança (OCon) e Orientação à Compreensão (OCom) - avaliado pela Escala Balbinotti-Cardoso de Suporte Social de Treinadores (EB-CSST-08), através de princípios métricos relativos às análises fatoriais e à consistência interna. Participaram do estudo 528 atletas de 13 a 19 anos ( $\bar{x}=$ $15,12 \pm 2,06)$. Os resultados das análises fatoriais exploratória (explicando 57,23\% da variância total) e confirmatória (GFI $=0,98 ; \mathrm{AGFI}=0,96 ; \mathrm{SRMR}=0,04 ; \chi^{2} / \mathrm{gl}=1,01 ; \mathrm{RMSEA}=$ $0,04 ; \mathrm{CFI}=0,99 ; \mathrm{NNFI}=0,98)$ permitem concluir que o modelo testado explorou e confirmou satisfatoriamente a existência de duas dimensões. Ainda, os resultados de fidedignidade $(\alpha=0,81 ; \omega=0,81 ;$ GLB $=0,88)$ asseguraram a precisão do modelo testado.
\end{abstract}

Palavras-chave: Validade. Confiabilidade. Liderança. Suporte social.

\section{Introdução}

O suporte social de treinadores tem se mostrado uma importante dimensão estudada em pesquisas recentes (NOCE et al., 2013; MITCHELL et al., 2014; DELL ANTONIO; BARROSO, 2014; MATIAS et al., 2014; ARNOLD; FLETCHER; ANDERSON, 2015; HØIGAARD et al., 2015; TOBAR, 2015; APPLETON; DUDA, 2016; SCHNAIDER et al., 2016; DUARTE; TEQUES; SILVA, 2017). Nesta pesquisa, visa-se apresentar as primeiras evidências de validade baseadas na estrutura interna e na precisão de um novo instrumento de medida desta dimensão: a Escala Balbinotti-Cardoso de Suporte Social de Treinadores (EBCSST-08). Esta é a segunda das cinco escalas que estão sendo elaboradas e que integrarão a Bateria Balbinotti-Cardoso de Comportamento de Liderança de Treinadores no contexto esportivo. 
Considerando a classificação compreensiva e descritiva da satisfação de atletas no esporte realizada por Chelladurai e Riemer (1997), cinco dimensões foram encontradas a partir do desenvolvimento do Questionário de Satisfação do Atleta (Athlete Satisfaction Questionnaire - SAQ; Riemer e Chelladurai, 1998). As cinco dimensões resultantes desta validação foram: a) a performance; b) a liderança do treinador; c) a equipe; d) o sistema organizacional, e, por fim, e) dimensão individual. Neste artigo, focalizaremos na dimensão liderança e, mais especificamente, sua dimensão Suporte Social. Para Chelladurai, Kuga e O'Bryant (1999), liderança significa todo o processo comportamental que tem por objetivo influenciar os membros de um grupo para trabalhar em direção aos objetivos comuns do mesmo grupo. Objetivando o trabalho com treinadores, Chelladurai e Saleh (1980) propuseram um Modelo de Liderança no Esporte, desenvolvendo a Escala de Liderança para o Esporte (Leadership Scale for Sport - LSS). Esta escala é ainda hoje utilizada em pesquisas neste tema (CHIU; RODRIGUEZ; WON, 2016; DUARTE; TEQUES; SILVA, 2017), e compreende cinco dimensões do comportamento de liderança: Treino e Instrução (TI), Comportamento Democrático (CD), Comportamento Autocrático (CA), Suporte Social (SS) e Feedback Positivo (FP). A dimensão SS é caracterizada por comportamentos de liderança do treinador que denotam uma preocupação pelo bem-estar de cada atleta, pela relação interpessoal calorosa e pela preocupação em estabelecer uma atmosfera de grupo positiva (Chelladurai, 1999).

Segundo Jowett e Poczwardowski (2007), Freeman e Rees (2010), Rees et al. (2012) e Weinberg e Gould (2016), a dimensão Suporte Social do Treinador (SST) impacta positivamente a saúde física e mental de seus atletas. As relações interpessoais são mais próximas, e o treinador não evita demonstrar amizade e afeição, refletindo diretamente na autoconfiança dos seus atletas. Jowett e Poczwardowski (2007) e Weinberg e Gould (2016) mencionam que os treinadores desempenham um papel fundamental sobre as experiências dos atletas e suas interações, oferecendo apoio e orientação, estabelecendo uma formação com ajuda pessoal, percebendo as suas necessidades e sendo compreensíveis nas relações interpessoais que, por sua vez, têm um impacto na motivação e no desenvolvimento de competências.

Diversos autores (HOSHINO; SONOO; VIEIRA, 2007; ANDREW, 2009; CRUZ; GOMES, 2009; ALBA; TOIGO; BARCELLOS; 2010; FREEMAN; REES, 2010; THOITS, 2011; THON et al., 2012; NOCE et al., 2013; DELL ANTONIO; BARROSO, 2014; WEINBERG; GOULD, 2016) têm mencionado (embora não tenha sido testado sistematicamente) a importância intrínseca de duas orientações fundamentais e inerentes à dimensão SST, sendo elas: Orientação à Confiança (OCon) e Orientação à Compreensão (OCom). Segundo Jowett e Poczwardowski (2007), Freeman e Rees (2010), Rees et al. (2012) e Weinberg e Gould (2016), o SST orientado à confiança se caracteriza pelo interesse no bemestar dos atletas, em manter um ambiente positivo de confiança mutua, de compromisso e convívio agradável, impactando, assim, positivamente na saúde física e mental de seus atletas. Nesta orientação, as relações interpessoais são mais próximas, nas quais o treinador demonstra amizade e afeição, refletindo diretamente na autoconfiança dos seus atletas e revelando, assim, um viés importante da dimensão OCon. Quanto à dimensão OCom, Gomes e Cruz (2006), Jowett e Poczwardowski (2007) e Weinberg e Gould (2016) mencionam que os treinadores têm grande influência nas experiências dos atletas e nas relações interpessoais, oferecem apoio e orientação, compreendendo e ajudando seus atletas em suas necessidades, na resolução de problemas e nas relações com seus colegas de equipe. O efeito do SST nessa orientação pode ser verificado no aumento do nível de motivação, na coesão da equipe, no desenvolvimento psicológico e no sentimento de competências dos atletas.

Considerando os estudos sobre comportamentos de liderança de treinadores, parece não existir escalas que avaliem precisamente, e de forma independente, o SST, permitindo assim uma exploração e uma confirmação de suas subdimensões latentes. Mesmo quando se analisa a escala proposta originalmente por Chelladurai e Saleh (1978), nota-se que esta não 
visa explorar as dimensões intrínsecas desse comportamento, revelando, assim, por este viés, a importância de ter uma escala independente de medida desse comportamento. Para tanto, necessita-se explorar alguns elementos empíricos, os quais darão suporte à escala em questão (ROCHETTE; BALBINOTTI, 2016).

Então, no plano empírico, alguns estudos têm demonstrado a importância capital do suporte social de treinadores, na relação com seus atletas. Alba, Toigo e Barcellos (2010), Prati e Pietrantoni (2013), Hampson e Jowett (2014) e Nogic (2014) concluíram que a variável SST tem sido associada à satisfação e ao rendimento de atletas, isto é, quanto maior a percepção positiva sobre essa dimensão, maior é a congruência entre a percepção dos treinadores e a dos atletas, resultando em uma melhoria no desempenho da tarefa e em um aumento na eficácia coletiva. Já Noce et al. (2013), Dell Antonio e Barroso (2014), Matias et al. (2014), Schnaider et al. (2016) e Tobar (2015) demonstraram que a variável SST se diferencia estatisticamente das variáveis treino e instrução, comportamento democrático e reforço positivo, concluindo tratar-se de uma variável, embora relacionada a estas, independente. Entre outros, Serpa (1990), Gomes, Pereira e Pinheiro (2008), Sonoo, Hoshino e Vieira (2008) e Nogic (2014) indicam que a variável SST de treinadores se diferencia em relação à idade e à maturidade dos atletas. A preferência por comportamentos de suporte social de treinador é maior nos mais jovens e mais impulsivos, em comparação com a preferência dos mais velhos e de maturação cognitiva maior. Ainda, para esses mesmos autores, essa preferência pode ser justificada pelos atletas das categorias de base pelo fato de ainda não estarem preparados psicologicamente para enfrentar fortes pressões emocionais.

Tendo como base o problema desta pesquisa (estudar a estrutura interna e a precisão da EB-CSST-08), os aspectos teóricos e empíricos supracitados, bem como sua importância no contexto esportivo (relação atleta-treinador) e as orientações e os estilos de liderança associados ao rendimento e à satisfação de atletas, foi possível elaborar as seguintes questões norteadoras desta pesquisa, sendo elas:

1) Quantos e quais são os fatores intrínsecos (ou dimensões latentes) à variável Suporte Social de Treinadores (SST), quando avaliados pela Escala BalbinottiCardoso de Suporte Social de Treinadores (EB-CSST-08)?

2) O modelo bidimensional inerente à EB-CSST-08 se adequa aos dados disponíveis?

3) Cada uma das dimensões avaliadas pela EB-CSST-08 é adequadamente precisa em sua medida, de forma que se possa confiar em seus resultados?

Para respondê-las adequadamente são empregados procedimentos éticos, metodológicos e estatísticos que serão apresentados a seguir.

\section{Procedimentos éticos, metodológicos e estatísticos}

O Comitê de Ética em Pesquisa da Universidade Federal do Rio Grande do Sul analisou e aprovou - Parecer $n^{\circ}$ : 1.435.916 - esta pesquisa. Participaram 528 atletas de seis clubes esportivos do estado do Rio Grande do Sul (os clubes não serão nomeados, conforme o pedido formal deles), das seguintes modalidades: futebol $=146$; judô $=28$; atletismo $=54$; voleibol $=72$; ginástica rítmica $=15$; ginástica artística $=12$; natação $=51$; basquetebol $=78$; handebol $=27$; futsal $=22$; esgrima $=23$, com idades variando de 13 a 19 anos $(\bar{x}=15,12 \pm 2,06)$. Todos foram assim classificados como "atletas jovens" (teenagers), segundo a proposição teórica sobre o desenvolvimento humano de Feldman (2016). Destaca-se que esta amostra foi dividida em duas subamostras, independentes $\left(n_{1}=264 ; n_{2}=264\right)$, com pareamento relativo por sexo $($ masculino $=388$; feminino $=140)$, sendo 194 atletas do sexo masculino e 70 atletas do sexo feminino, por subamostra. A fim de melhor descrever a amostra total, cabe salientar que todos os atletas eram inscritos em suas respectivas federações e, considerando suas idades 
e o censo do IBGE (2010), estima-se que, em sua grande maioria, eram estudantes de ensino básico $(n=448)$.

O recrutamento dos atletas se deu observando os seguintes passos: (1) os pesquisadores contataram os dirigentes técnicos e esportivos dos seis clubes pesquisados (destaca-se que todos os seis clubes concordaram em participar da pesquisa); (2) com a acessibilidade garantida, foram fixados cartazes-convites nas dependências dos clubes e foi pedido para os treinadores reforçarem o convite; (3) os atletas menores de idade, que se apresentaram em local e na hora predeterminados (nas dependências dos clubes), já trouxeram o termo de assentimento assinado pelos seus responsáveis; (4) foi-lhes dito, mais uma vez, que todos eram convidados (não obrigados) a participar e que podiam retirar seus nomes a qualquer momento (presente ou futuro) da pesquisa, sendo, para isto, apenas necessário contatar os pesquisadores (não houve desistências), e, (5) com a concordância estabelecida, os treinadores (sempre presentes durante a aplicação do questionário) assinaram os termos de consentimento e a aplicação do EB-CSST-08 começou. Trata-se, então, de uma amostra não aleatória recomendada para estudos em educação e psicologia (MAGUIRE; ROGERS, 1989).

Os dois instrumentos utilizados nesta pesquisa foram: o Questionário Biosociodemográfico, apenas para o controle das variáveis Sexo, Idade e Modalidade, e a Escala Balbinotti-Cardoso de Suporte Social de Treinadores (EB-CSST-08). A EB-CSST-08 (Balbinotti; Cardoso, 2016) foi elaborada com o objetivo de poder conhecer melhor a estrutura subjacente do construto Suporte Social de Treinadores (SST), no contexto brasileiro. Os oito itens que integram essa escala (ver a escala anexa) foram elaborados com base no Modelo Multidimensional de Liderança no Esporte, proposto por Chelladuarai e Saleh (1978), e foram avaliados por um comitê de cinco juízes colaboradores (dois doutores em Psicologia do Esporte, dois doutores em Ciências do Movimento Humano, e um treinador com 27 anos de experiência com jovens atletas), quanto à clareza e à pertinência (CASSEPP-BORGES; BALBINOTTI; TEODORO, 2010) de cada um dos itens. Os resultados $\left(\mathrm{CVC}_{t, i, c}>0,80\right)$ dos cálculos de validade de conteúdo do EB-CSST-08 indicaram a clareza e a pertinência do conteúdo dos itens na amostra em questão.

$\mathrm{O}$ atleta responde a cada item de acordo com uma escala bidirecional, de tipo Likert, graduada em cinco pontos, indo de "discordo fortemente" (1) a "concordo fortemente" (5) com a assertiva. $\mathrm{O}$ escore total (e por dimensão) é obtido somando-se os pontos acordados pelo atleta. Quanto maior for o escore obtido, maior será o nível de concordância (preferência) do atleta com os conteúdos da dimensão SST, podendo-se interpretá-la, pelo viés teórico (JOWETT; POCZWARDOWSKI, 2007; FREEMAN; REES, 2010; REES et al., 2012; WEINBERG; GOULD, 2016), como favorecendo o desempenho e a satisfação, resultando em um impacto positivo na saúde física e mental dos atletas.

Os procedimentos estatísticos estão baseados em análises fatoriais exploratória e confirmatória (BROWN, 2016; KLINE, 2015; ROCHETTE; BALBINOTTI, 2016), com subamostras independentes para cada uma destas análises $\left(n_{1}=264 ; n_{2}=264\right)$, assim como cálculos de consistência interna (CRONBACH; SHAVELSON, 2004; KLINE, 2015), sendo todas as análises realizadas com base em matrizes policóricas (MUTHÉN; KAPLAN, 1992).

\section{Resultados, interpretações e discussões}

A fim de responder, adequadamente, às três questões centrais desta pesquisa, procedeu-se à exploração dos escores obtidos pela EB-CSST-08, segundo princípios norteadores comumente aceitos na literatura especializada (BROWN, 2016; BYRNE, 2016; CRONK, 2016; FINCH; IMMEKUS; FRENCH, 2016; MEYERS; GAMST; GUARINO, 2016; PRICE, 2016; ROCHETTE; BALBINOTTI, 2016). Salienta-se que as análises fatoriais (exploratória e con- 
firmatória) e os cálculos de consistência interna ( $\alpha, \omega, \mathrm{GLB}$, Precisão) estão apresentados de forma sucessiva e sistemática.

\section{Análises fatoriais exploratória e confirmatória}

Para que se possa responder à primeira das três questões centrais desta pesquisa (quantos e quais são os fatores intrínsecos à variável Suporte Social de treinadores, quando avaliados pela EB-CSST-08?), efetuou-se uma análise fatorial exploratória $\left(n_{1}=286\right)$. Para garantir a adequada interpretação dessa análise, foram estimados os seguintes coeficientes: (1) KaiserMeyer-Olkin (KMO = 0,79); (2) Determinante de Matriz de Correlação (0,23), e (3) Teste de Esfericidade de Bartlet $\left(\chi_{(28)}^{2}=384,1 ; p<0,01\right)$. Seus resultados indicam que as correlações entre os itens são suficientes para se proceder com as análises fatoriais exploratória e confirmatória. $\mathrm{O}$ resultado da medida de redundância de informação $(|\mathrm{R}|$ diferente de 0$)$ indica a ausência de todo tipo de repetição das ligações correlativas lineares (FIELD, 2016; ROCHETTE; BALBINOTTI, 2016), assegurando a pertinência do uso de cálculos fatoriais.

Assim, uma análise Robust Diagonally Weighted Least Squares (RDWLS), seguida de uma rotação oblíqua (promax), serviu para examinar a estrutura fatorial exploratória da EBCSST-08 (ver Tabela 1). A partir desta análise, extraíram-se dois fatores com raízes latentes superiores a um (1). Eles explicam 57,23\% da variância total do construto. Considerando que as comunalidades dos itens são todas superiores a 0,37 , esta solução mostra-se satisfatória, apresentando-se de forma pura (ver Tabela 1) com itens saturando de forma importante $\left(\right.$ Sat $_{f} \geq$ 0,37 ) em seus determinados fatores de origem.

Após ter-se determinado a bidimensionalidade da variável SS (quando avaliada pela EB-CSST-08), resta determinar quais são estes fatores. Uma das possíveis formas é nomeálos (ROCHETTE; BALBINOTTI, 2016). Mesmo sabendo que o nome dos fatores é uma escolha dos autores (ver Tabela 1), portanto um procedimento fundamentalmente qualitativo (HUTZ; BANDEIRA; TRENTINI, 2015; ROCHETTE; BALBINOTTI, 2016), efetuaram-se cálculos Kappa de Cohen $(\mathrm{k}=0,98)$, com quatro juízes colaboradores, e seus resultados confirmam a adequabilidade dos nomes dos fatores.

As comunalidades $\left(h^{2}\right)$ e as saturações fatoriais exploratórias confirmam que o modelo avaliado pela EB-CSST-08 é de estrutura bidimensional. As duas orientações obtidas (Orientação à Confiança e Orientação à Compreensão) deixam claro que o SST não deve ser reduzido a uma interpretação simples (unidimensional).

Tabela 1. Análise fatorial dos resultados obtidos com o EB-CSST-08 em atletas de ambos os sexos e de diferentes modalidades esportivas $\left(n_{1}=264\right)$

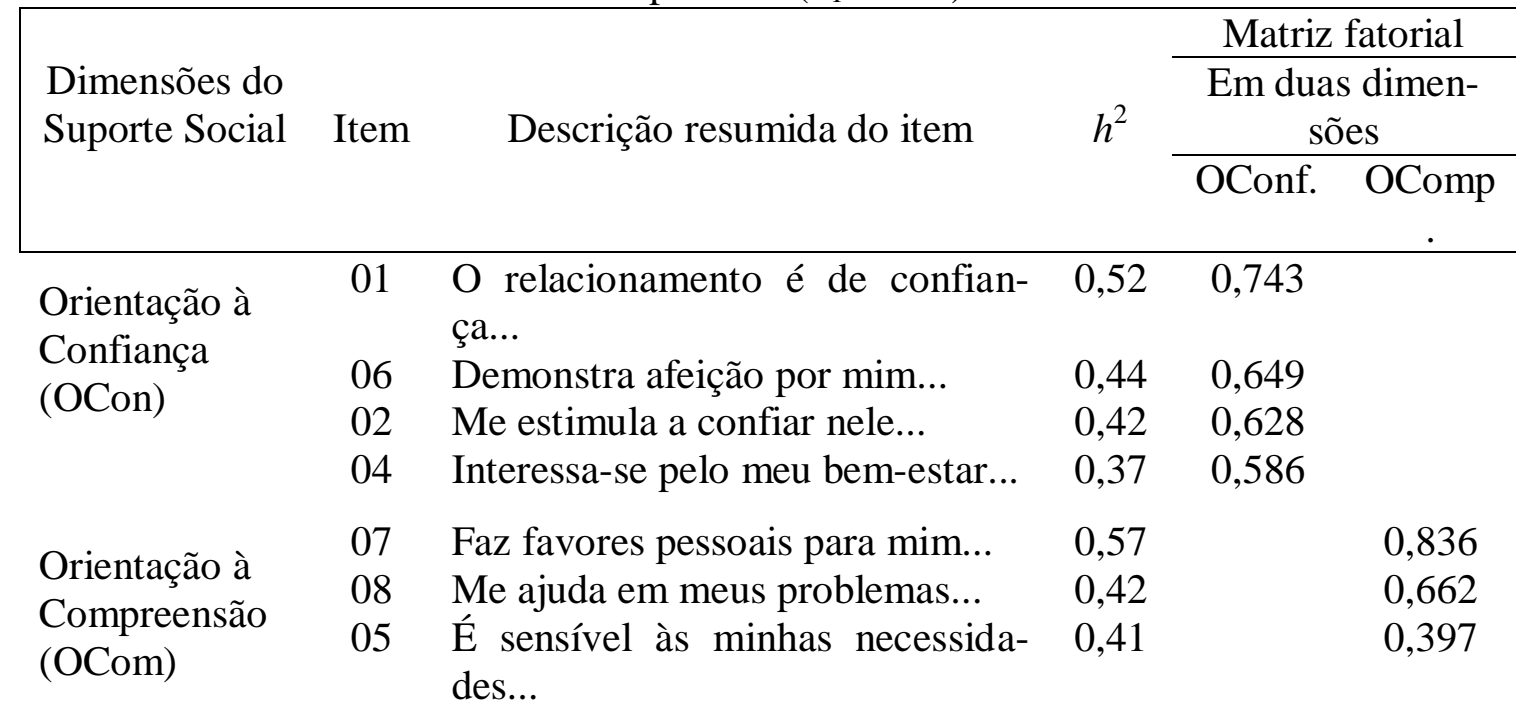


03 Me ajuda nas relações com os 0,37

0,367

atletas

\begin{tabular}{lrrc}
\hline Porcentagem da variância por fator - valores extraídos & & 42,54 & 14,69 \\
\hline & $\begin{array}{r}\text { To- } \\
\text { tal }\end{array}$ & OCon & OComp \\
\cline { 2 - 4 } & 0,81 & 0,76 & 0,71 \\
Alpha de Cronbach (1951) & 0,81 & 0,76 & 0,71 \\
Ômega de McDonald (1999) & 0,88 & 0,78 & 0,74 \\
GLB (Greatest Lower Bound) Woodhouse (1977) & 0,82 & 0,77 & 0,71 \\
Precisão de Mislevy \& Bock (1990) &
\end{tabular}

Obs.: Método de extração: RDWLS (Robust Diagonally Weighted Least Squares)

Rotação convergida em cinco interações

Fonte: Os autores, 2017

A variável SST, quando medida pela EB-CSST-08, não deve ser unicamente interpretável em sentido geral, tal como: "este atleta avalia seu treinador como uma pessoa que lhe dá suporte social". Observações/interpretações como esta podem, no mínimo, representar uma falta de precisão, sendo mais adequado especificar a orientação de que se está falando. Pois, a partir de uma afirmação geral, deixa-se de formular importantes questionamentos que devem ser particularmente interessantes aos pedagogos do esporte, treinadores e mesmo outros responsáveis pelo desenvolvimento de atletas, tais como: qual orientação adotada pelo treinador que influencia mais as características competitivas dos atletas? Qual a relação entre as orientações e a satisfação dos atletas? Questões como estas são importantes na medida em que pedagogos e psicólogos do esporte, assim como treinadores, queiram, por exemplo, analisar o rendimento e a satisfação dos atletas, levando-se em consideração os diferentes contextos, objetivos e características que lhes são próprias.

Holt e Hoar (2006), Freeman e Rees (2010), Rees et al. (2012) e Weinberg e Gould (2016) afirmam que a dimensão SST é derivada das relações interpessoais entre treinador e atleta, com a finalidade de melhorar o bem-estar do atleta que, por sua vez, identifica esse apoio como um importante recurso motivacional para sua prática, refletindo no aumento de sua autoconfiança e seu desempenho. Ainda, Thoits (2011), Sullivan et al. (2012) e Weinberg e Gould (2016) referem que o suporte social, dado pelo treinador ao atleta, impacta diretamente o desenvolvimento pessoal e social dos esportistas. Para estes autores, o desenvolvimento positivo dos atletas relaciona-se ao comportamento de treinadores, cuja orientação é focada no sujeito, em sua maestria, estabelecendo, assim, um clima de amizade e confiança mutua. A dimensão SST apresenta relações com outras variáveis psicossociais como a autoconfiança, o estresse e a autoeficácia (COSTA; SAMULSKI; COSTA, 2009; FREEMAN; REES, 2010; WEINBERG; GOULD, 2016). Segundo Malinauskas (2010), o estresse, quando atinge níveis muito elevados, apresenta prejuízos para a saúde mental, o bem-estar e o rendimento dos atletas. Em contrapartida, os atletas que recebem mais suporte social de seu treinador apresentam níveis mais baixos de estresse, melhorando o seu desempenho na tarefa. Os resultados evidenciados em diversos estudos (GOMES; PEREIRA; PINHEIRO, 2008; HOSHINO; SONOO; VIEIRA, 2007; ANDREW, 2009; ALBA; TOIGO; BARCELLOS, 2010; NOGIC, 2014), com diferentes modalidades esportivas, sugerem que um ambiente positivo de liderança do treinador, com suporte social, aumenta a satisfação e o rendimento individual dos atletas, principalmente nas modalidades individuais, podendo ser explicados pela maior proximidade nas relações e pela diminuição nas pressões em atingir metas e objetivos, de responsabilidade individual.

Para que se possa responder à segunda das três questões centrais desta pesquisa (o modelo bidimensional inerente à EB-CSST-08 se adequa aos dados disponíveis?), efetuou-se uma análise fatorial confirmatória, com uma amostra independente $\left(n_{2}=264\right)$ e com parea- 
mento relativo por sexo e idade. Esta foi calculada, e seus resultados estão apresentados de acordo com as indicações de Brown (2016) e Kline (2015) (ver Tabela 2), os quais recomendam a apresentação de, no mínimo: um índice relativo ao ajustamento absoluto do modelo, o qual permite avaliar o quanto a matriz de variância-covariância observada é estatisticamente similar à matriz estimada (MCDONALD; HO, 2002; HOOPER; COUGHLAN; MULLEN, 2008); um índice relativo à correção parcimoniosa (similar ao anterior, porém integra um procedimento estatístico que permite corrigir um possível mau ajustamento absoluto inicial do modelo - KALINOWSKI; WAGNER; TAPER, 2006), e um índice de ajustamento comparativo, o qual permite avaliar o ajustamento do modelo hipotético pelo viés do modelo nulo, isto é, com covariâncias iguais a zero (GATIGNON, 2010). A Tabela 2 apresenta os resultados pertinentes.

Tabela 2. Resultados da análise fatorial confirmatória da EB-CSST-08 $\left(n_{2}=264\right)$

Índices de ajustamento absoluto

\begin{tabular}{ccc}
$\begin{array}{c}\text { Índice de } \\
\text { correlação } \\
\text { parcimoni- } \\
\text { osa }\end{array}$ & $\begin{array}{c}\text { Índices de ajus- } \\
\text { tamento compara- } \\
\text { tivo }\end{array}$ \\
RMSEA & NNFI & CFI \\
0,04 & 0,98 & 0,99 \\
\hline
\end{tabular}

Obs.: * ${ }^{*}>0,05$

Fonte: Os autores, 2017

Considerando que o resultado do qui-quadrado não foi significativo ( $p>0,05)$, é evidente que sua relação com o grau de liberdade (qui-quadrado normalizado) apresenta-se satisfatória $\left(\chi^{2} / \mathrm{gl}<2,0\right)$. Os demais resultados relativos aos índices de ajustamento absoluto também se mostraram satisfatórios (GFI > 0,95; AGFI > 0,95; SRMR < 0,05), indicando que os dados se adequam satisfatoriamente, e sem exceções, ao modelo hipotético e à matriz de covariância (HOOPER; COUGHLAN; MULLEN, 2008; KLINE, 2015), não sendo necessária a interpretação do índice parcimonioso (RMSEA < 0,05), que de qualquer forma também foi satisfatório. Por fim, pode-se afirmar que os índices de ajustamento comparativo também se mostraram satisfatórios (CFI > 0,95), demonstrando uma proximidade de ajustamento do modelo, ao examinar a diferença entre os dados disponíveis e o modelo hipotético, levando-se em consideração o tamanho da amostra inerente ao teste do qui-quadrado de ajustamento do modelo. A Figura 1 apresenta a ilustração dos resultados do modelo estrutural propriamente dito. 


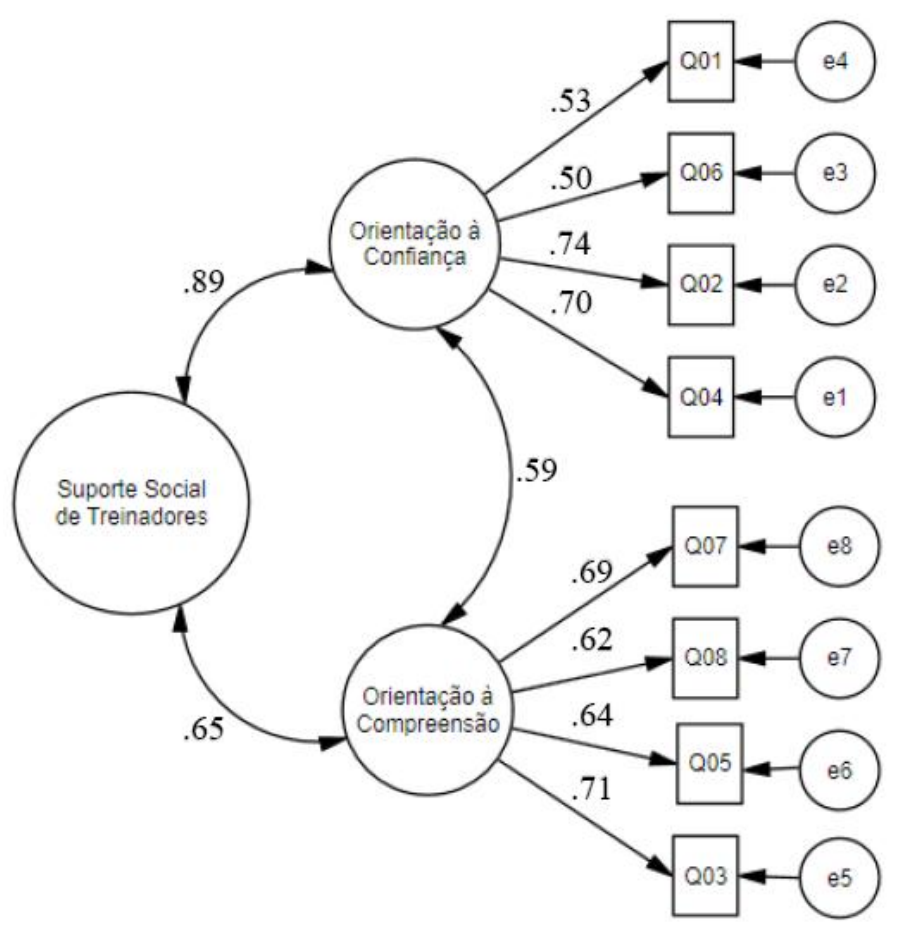

Figura 1 - Diagrama de caminhos e relações da percepção de atletas sobre o suporte social de treinadores em contexto esportivo

Fonte: Os autores, 2017

A correlação entre os dois fatores pode ser classificada como forte, positiva e, considerando a análise das covariâncias, significativa $(\mathrm{p}<0,05)$. O resultado do coeficiente de determinação foi cerca de $35 \%$, indicando que $35 \%$ da variância da variável OCon é explicada pela variância da variável OCom. Estes resultados indicam que os fatores em questão, por compartilharem uma parte moderada de variância comum, integram um fator geral, de segunda ordem, o qual pode ser caracterizado pelo construto em estudo (Suporte Social de Treinadores). Os índices relativos às regressões estandardizadas variaram, independentemente do fator, entre 0,50 e 0,74 , indicando que as correlações múltiplas quadradas são todas satisfatórias $(0,25$ $\left.\leq R^{2} \geq 0,55\right)$, pois estes resultados são iguais ou maiores que a convenção aceita em estudos fatoriais (ROCHETTE; BALBINOTTI, 2016). Todos estes resultados não deixam dúvidas quanto à interpretação positiva das evidências de validade, pelo viés da estrutura interna, do modelo bidimensional da EB-CSST-08, conforme os dados empíricos disponíveis. Em uma interpretação de caráter mais geral desses dados, pode-se inferir que o funcionamento cognitivo do grupo de atletas pesquisados, quando requeridos a responder sobre as suas próprias percepções relativas ao suporte social de seus treinadores, pode ser compartimentalizado em duas dimensões distintas, mas relacionáveis. Destaca-se, então, que esse modelo bidimensional foi confirmado, respondendo, positivamente, à segunda questão central desta pesquisa.

\section{Cálculos de Consistência Interna}

Para que se possa responder à terceira das três questões centrais desta pesquisa (cada uma das dimensões avaliadas pela EB-CSST-08 é adequadamente precisa em sua medida, de forma que se possa confiar em seus resultados?), efetuaram-se cálculos de consistência interna (Alpha de Cronbrach, Ômega de McDonald, GLB de Woodhouse e Precisão de Mislevy \& Bock), baseados em matrizes policóricas. Todos os resultados destas análises de consistência interna (ver Tabela 1) variam de 0,71 a 0,88. Tais resultados são indicadores satisfatórios de precisão da escala total (SST) e de cada uma das duas dimensões (OCon e OCom), podendo- 
se dizer que os resultados obtidos em cada um dos quatro itens de cada dimensão são consistentes entre si, representando uma medida precisa do construto e de suas dimensões.

\section{Conclusões, limitações e perspectivas para novos estudos}

Este trabalho permitiu demonstrar o modelo bidimensional avaliado pela EB-CSST08, ao mesmo tempo em que se demonstraram as nuances do conceito de SST, através de dados empíricos, coletados no contexto brasileiro. As duas orientações do construto SST, quando avaliadas pela EB-CSST-08, contribuem para o conhecimento da área da Psicologia e da Pedagogia do Esporte, no sentido de aperfeiçoar a interpretação/classificação desta que é uma das dimensões do Comportamento de Liderança no Esporte. Com base nos resultados deste estudo, verificamos a ampliação da dimensão SST, passando de uma medida unidimensional, conforme a estrutura hierárquica do modelo Multidimensional de Liderança no Esporte, difundido por Chelladurai e Saleh (1978), para uma medida bidimensional, sendo: orientada à confiança (relacionamento próximo de amizade e confiança, voltado ao bem-estar do atleta) e orientada à compreensão (o treinador oferece apoio e orientação, compreendendo e ajudando seus atletas em suas necessidades, na resolução de problemas e nas relações com seus colegas de equipe).

As limitações de caráter amostral (amostra por conveniência e diversidade de categorias e modalidades esportivas) não permitem a generalização dos resultados à população de atletas, entretanto, considerando o $n$ amostral não desprezível $(n=528)$, acredita-se que os resultados obtidos são fortes indicadores da realidade, podendo-se concluir que a escala EBCSST-08 apresenta fortes evidências de validade pelo viés de sua estrutura interna e precisão. Finaliza-se este estudo indicando que estes resultados e conclusões podem ser particularmente úteis para psicólogos e pedagogos do esporte, treinadores e mesmo outros profissionais interessados no desenvolvimento de modelos que expliquem o fenômeno do suporte social de treinadores no contexto esportivo, num sentido mais específico e, inclusive, em modelos de treino e competição, num sentido mais amplo. Portanto, elementos como "Orientação à Confiança" (OCon) e "Orientação às Compreensão" (OCom) - dimensões avaliadas pela EBCSST-08 - parecem ser uma fonte importante de informação referente ao rendimento e à satisfação dos atletas (incidindo, portanto, e conforme o conteúdo teórico e empírico apresentado, na saúde mental e física dos atletas), permitindo que esses profissionais entendam melhor como esses elementos se integram na dinâmica geral das relações treinador-atleta. Destaca-se que estes profissionais devem considerar os resultados aqui apresentados para integrá-los em seus comportamentos, atitudes e relações com os atletas, tanto nas situações de treino quanto nas competições, principalmente, por estarem relacionados com o bem-estar e o desempenho destes atletas.

Por fim, cabe salientar que outras avaliações (autoconceito, autoestima, motivação, autodeterminação, estresse, interesses etc.) são particularmente interessantes quando utilizadas dentro de um contexto maior, principalmente, quando esses profissionais se interessam em ajudar o atleta em sua dinâmica geral de funcionamento psicopedagógico e esportivo. Finalizando, novos estudos podem ser conduzidos com o objetivo de responder a outras importantes questões de natureza psicométrica, tais como a estabilidade temporal do modelo testado (fidedignidade teste-reteste), a validade de critério (SST versus satisfação no esporte; validações cruzadas), a invariância do modelo testado (categorias esportivas, sexo, estado civil etc.), entre diversas outras, as quais não fizeram parte do escopo desta pesquisa. 


\title{
STUDY OF INTERNAL STRUCTURE AND PRECISION OF SCALE BALBINOTTI- CARDOSO THE SOCIAL SUPPORT OF COACHES (EB-CSST-08)
}

\begin{abstract}
The objective of this study is to test the two-dimensional model - guidance to the Trust (OCon) and guidance to understanding (OCom) - evaluated by the Balbinotti-Cardoso scale of Social support of coaches (EB-CSST-08), through metric principles relating to the analyses factorials and Internal consistency. Participated in the study 528 athletes from 13 to 19 years $(\bar{x}=15,12 \pm 2,06)$.The results of factorials exploratory analyses (explaining $57.23 \%$ of total variance) and confirmatory $\left(\mathrm{GFI}=0,98 ; \mathrm{AGFI}=0,96 ; \mathrm{SRMR}=0,04 ; \chi^{2} / \mathrm{gl}=1,01 ; \mathrm{RMSEA}=\right.$ 0,$04 ; \mathrm{CFI}=0,99 ; \mathrm{NNFI}=0,98$ ) allow you to conclude that the tested model has explored and satisfactorily confirmed the existence of two dimensions. Still, the results of trust $(\alpha=0,81 ; \omega$ $=0,81 ;$ GLB $=0,88$ ) assured the accuracy of the tested model.
\end{abstract}

Key words: Validity. Reliability. Leadership. Social support.

\section{ESTUDIO DE LA ESTRUCTURA INTERNA Y PRECISIÓN DE LA ESCALA BAL- BINOTTI-CARDOSO DE APOYO SOCIAL DE ENTRENADORES (EB-CSST-08)}

\begin{abstract}
Resumen
El objetivo de este estudio es probar el modelo bidimensional - Orientación a la Confianza (OCon) y Orientación a la Comprensión (eran alguien) - evaluados por la escala BalbinottiCardoso de apoyo social de los coaches (EB-CSST-08), a través de principios métricos relacionados con los análisis factoriales y Consistencia interna. Participó en el estudio 528 atletas de 13 a 19 años $(\bar{x}=15,12 \pm 2,06)$. Los resultados de los análisis factoriales exploratorios (explicando el 57,23\% de la varianza total) y confirmativos $(\mathrm{GFI}=0,98$; AGFI $=0,96$; SRMR $=$ 0,$\left.04 ; \chi^{2} / \mathrm{gl}=1,01 ; \mathrm{RMSEA}=0,04 ; \mathrm{CFI}=0,99 ; \mathrm{NNFI}=0,98\right)$ le permiten concluir que el modelo probado ha explorado y confirmado satisfactoriamente la existencia de dos dimensiones. Aun así, los resultados de la confianza $(\alpha=0,81 ; \omega=0,81 ; \mathrm{GLB}=0,88)$ aseguró la exactitud del modelo probado.
\end{abstract}

Palabras clave: Validez. Fiabilidad. Liderazgo. Apoyo social.

\section{Referências}

ALBA, G. D. R.; TOIGO, T.; BARCELlOS, P. F. P. Percepção de atletas profissionais de basquetebol sobre o estilo de liderança do técnico. Revista Brasileira de Ciências do Esporte, v. 32, n. 1, p. 143-159, 2010.

ANDREW, D. P. The impact of leadership behavior on satisfaction of college tennis players: a test of the leadership behavior congruency hypothesis of the multidimensional model of leadership. Journal of Sport Behavior, v. 32, n. 3, p. 261-277, 2009.

APPLETON, P. R.; DUDA, J. L. Examining the interactive effects of coach-created empowering and disempowering climate dimensions on athletes' health and functioning. Psychology of Sport and Exercise, v. 26, p. 61-70, 2016.

ARNOLD, R.; FLETCHER, D.; ANDERSON, R. Leadership and management in elite sport: factors perceived to influence performance. International Journal of Sports Science \& Coaching, v. 10, n. 2-3, p. 285-304, jun. 2015. 
BROWN, T. A. Confirmatory factor analysis for applied research. 2. ed. New York: Guilford Publications, 2016.

BYRNE, B. M. Structural equation modeling with AMOS: basic concepts, applications, and programming. 3. ed. New York: Routledge, 2016.

CASSEPP-BORGES, V.; BALBINOTTI, M. A. A.; TEODORO, M. L. M. Tradução e validação de conteúdo: uma proposta para a adaptação de instrumentos. In: PASQUALI, L. (Ed.). Intrumentação psicológica: fundamentos e práticas. Porto Alegre: Artmed, 2010. p. 506-520.

CHELlADURAI, P.; SALEH, S. D. Preferred leadership in sports. Canadian Journal of Applied Sport Science, v. 3, 1978.

CHELLADURAI, P.; SALEH, S. D. Dimensions of leader behavior in sports: development of a leadership scale. Journal of Sport Psychology, v. 2, n. 1, p. 34-45, 1980.

CHELlADURAI, P. Leadership in sports: A review. International Journal of Sport Psychology, Italy, v. 21, n. 4, p. 328-354, 1990.

CHELLADURAI, P.; RIEMER, H. A classification of facets of athlete satisfaction. Journal of Sport Management, v. 11, n. 2, p. 133-159, 1997.

CHELLADURAI, P.; KUGA, D.; O'BRYANT, C. Individual differences, perceived task characteristics, and preferences for teaching and coaching. Research Quarterly For Exercise and Sport, v. 70, n. 2, p. 179-189, 1999.

CHIU W, RODRIGUEZ FM, WON D. Revisiting the leadership scale for sport examining factor structure through exploratory structural equation modeling. Psychological Reports, 2016; v. 119, n. 2, p. 435-49.

COSTA, I. T. D.; SAMULSKI, D. M.; COSTA, V. T. D. Análise do perfil de liderança dos treinadores das categorias de base do futebol brasileiro. Revista Brasileira de Educação Física Esporte, v. 23, n. 3, p. 185-194, 2009.

CRONBACH, L. J.; SHAVELSON, R. J. My current thoughts on coefficient alpha and successor procedures. Educational and Psychological Measurement, v. 64, n. 3, p. 391-418, 2004.

CRONK, B. C. How to use SPSS®: a step-by-step guide to analysis and interpretation. 9. ed. New York: Routledge, 2016.

CRUZ, T.; GOMES, G. Estilo de liderança esportiva: o impacto em equipes de handebol. In: ENCONTRO INTERNACIONAL DE CESUMAR. 2009. Anais..., Paraná: Centro Universitário de Maringá, 2009. p. 1-5.

DELL ANTONIO, E.; BARROSO, M. L. C. Perfil de liderança de treinadores e desempenho de equipes em competição. Aval. psicol, v. 13, n. 3, p. 311-316, 2014. 
DUARTE, D.; TEQUES, P.; SILVA, C. Liderança e satisfação no futebol: testagem da congruência com recurso a análise de equações estruturais. Revista Iberoamericana de Psicología del Ejercicio y el Deporte, v. 12, n. 1, p. 71-82, 2017.

FELDMAN R.S. Life span development: a topical approach. 3. ed: Boston, MA: Pearson, 2016.

FIELD, A. Discovering statistics using IBM SPSS statistics. 4. ed. London: Sage, 2016.

FINCH, W. H.; IMMEKUS, C. J.; FRENCH, B. F. Applied psychometrics using SPSS and AMOS. Charlotte, EUA: Age Publishing, 2016.

FLETCHER, R. B.; ROBERTS, M. H. Longitudinal stability of the leadership scale for sports. Measurement in Physical Education and Exercise Science, v. 17, n. 2, p. 89-104, 2013.

FREEMAN, P.; REES, T. Perceived social support from team-mates: direct and stressbuffering effects on self-confidence. European Journal of Sport Science, v. 10, n. 1, p. 5967, 2010.

GATIGNON, H. Reliability Alpha, principle component analysis, and exploratory factor analysis. In: (Ed.). Statistical analysis of management data. New York: Springer, 2010. p. 29-57.

GOMES, A. R.; CRUZ, J. F. Relação treinador-atleta e exercício da liderança no desporto: a percepção de treinadores de alta competição. Estudos de Psicologia, v. 11, n. 1, p. 5-15, 2006.

GOMES, A. R.; PEREIRA, A. P.; PINHEIRO, A. R. Liderança, coesão e satisfação em equipas desportivas: um estudo com atletas portugueses de futebol e futsal. Psicologia: Reflexão e Crítica, v. 21, n. 3, p. 482-491, 2008.

HAMPSON, R.; JOWETT, S. Effects of coach leadership and coach-athlete relationship on collective efficacy. Scandinavian Journal of Medicine and Science in Sports, v. 24, n. 2, p. 454-460, 2014.

HØIGAARD, H.; DE CUYPER, B.; FRANSEN, K.; BOEN, F.; PETERS, D. M. Perceived coach behavior in training and competition predicts collective efficacy in female elite handball players. International Journal of Sport Psychology, v. 46, p. 321-336, 2015.

HOLT, N. L. ; HOAR, S. D. The multidimensional construct of social support. Literature reviews in sport psychology, 2006. p. 199-225

HOOPER, D.; COUGHLAN, J.; MULLEN, M. Evaluating model fit: a synthesis of the structural equation modelling literature. In: 7 TH EUROPEAN CONFERENCE ON RESEARCH METHODOLOGY FOR BUSINESS AND MANAGEMENT STUDIES. Anais... London: Regent's College, 2008. p.195-200. Disponivel em: <http://arrow.dit.ie/cgi/viewcontent.cgi?article=1046\&context=buschmancon> Acesso em: 10 mar. 2017. 
HOSHINO, E. F.; SONOO, C. N.; VIEIRA, L. F. Perfil de liderança: uma análise no contexto esportivo de treinamento e competição. Revista da Educação Física/UEM, v. 18, n. 1, p. 7783, 2007.

HUTZ, C. S.; BANDEIRA, D. R.; TRENTINI, C. M. Psicometria. Porto Alegre: Artmed Editora, 2015.

JOWETT, S.; POCZWARDOWSKI, A. Understanding the coach-athlete relationship. In: JOWETT, S; LAVALLEE, D (Eds.) Social psychology in sport., Champaing IL, Human Kinetics. 2007. p. 3-14, Disponivel em: <https://www.researchgate.net/publication/232506356_Understanding the_CoachAthlete Relationship> Acesso: em 10 mar. 2017.

KALINOWSKI, S. T.; WAGNER, A. P.; TAPER, M. L. ML - Relate: a computer program for maximum likelihood estimation of relatedness and relationship. Molecular Ecology Notes, v. 6, n. 2, p. 576-579, 2006.

KLINE, R. B. Principles and practice of structural equation modeling. 3. ed., New York: Guilford publications, 2015.

MAGUIRE, T. O.; ROGERS, W. T. Proposed solutions for nonrandomness in educational research. Canadian Journal of Education/Revue canadienne de l'education, p. 170-181, 1989.

MALINAUSKAS, R. The associations among social support, stress, and life satisfaction as perceived by injured college athletes. Social Behavior and Personality: an international journal, v. 38, n. 6, p. 741-752, 2010.

MATIAS, T. S.; HINKEL, D.; GONÇALVES, E.; BERNARDO, V. M. Perfil de liderança de treinadores de triatlo do Brasil. Revista Portuguesa de Ciências do Desporto, 2014.

MCDONALD, R. P.; HO, M-H. R. Principles and practice in reporting structural equation analyses. Psychological Methods, v. 7, n. 1, p. 64, 2002.

MEYERS, L. S.; GAMST, G.; GUARINO, A. J. Applied multivariate research: design and interpretation. 3 ed. London: Sage, 2016.

MITCHELL, I.; EVANS, L.; REES. T.; HARDY, L. Stressors, social support, and tests of the buffering hypothesis: Effects on psychological responses of injured athletes. British Journal of Health Psychology, v. 19, n. 3, p. 486-508, 2014.

MUTHÉN, B.; KAPLAN, D. A comparison of some methodologies for the factor analysis of non - normal Likert variables: a note on the size of the model. British Journal of Mathematical and Statistical Psychology, v. 45, n. 1, p. 19-30, 1992.

NOCE, F.; TEIXEIRA T. M.; LOPES, M. C.; SAMULSKI, D. M.; SOUZA, P. R. C. Leadership of futsal coaches. Revista de Psicologia del Deporte, v. 22, n. 1, p. 11-17, 2013. Disponível em: < http://www.rpd-online.com/article/view/v22-n1-noce-de matos-calabriasamulski-coelho >. Acesso em: 12 mar. 2017. 
NOGIC, G. Estilo de liderança dos treinadores de basquetebol em Portugal. Perceção, preferência e nível de satisfação dos atletas da Liga Profissional Masculina. 2014. Dissertação (Mestrado) - Curso de Educação Física - Treinamento Desporto, Instituto Politécnico de Santarém, Escola Superior de Desporto de Rio Maior, 2014. Disponível em: < http://repositorio.ipsantarem.pt/bitstream/10400.15/1177/2/Disserta\%C3\%A7\%C3\%A3o\%20 MESTRADO\%20Goran\%20Nogic.pdf >. Acesso em: 5 mar. 2017.

PRATI, G.; PIETRANTONI, L. The effects of congruency and discrepancy of sport leadership behaviors on objective performance. International Journal of Sport Psychology, v. 44, n. 6, p. 546-564, 2013.

PRICE, L. R. Psychometric methods: theory into practice. New York: Guilford Publications, 2016.

REES, T.; FREEMAN. P.; BELL, S.; BUNNEY, R. Three generalizability studies of the components of perceived coach support. Journal of Sport and Exercise Psychology, v. 34, n. 2, p. 238-251, 2012.

RIEMER, H.; CHELlADURAI, P. Development of the athlete satisfaction questionnaire (ASQ). Journal of Sport \& Exercise Psychology, v. 20, n. 2, p. 127-156, jun. 1998.

ROCHETTE, S.; BALBINOTTI, M. A. A. Guidelines for using exploratory fator analysis to test constructo validity of inventories in sports research. American Journal of Sports Training, v. 3, 28 dez. 2016. Disponível em: < http://www.ajst.science/article/417/ >. Acesso em: 8 mar. 2017.

SCHNAIDER, G. B. ; BORGES , P. H.; BORGHI, G. A. B.; MAGOSSI, M. A. O.; OLIVEIRA, J. R.; RINALDI, W. Relação entre o perfil de liderança do treinador e a motivação em jovens jogadores de futebol. Pensar a Prática, v. 19, n. 4, 2016.

SERPA, S. O treinador como líder: panorama actual da investigação. Ludens, v. 12, n. 2, p. 23-32, 1990.

SONOO, C. N.; HOSHINO, E. F.; VIEIRA, L. F. Liderança esportiva: estudo da percepção de atletas e técnicos no contexto competitivo. Psicologia Teoria e Prática, v. 10, n. 2, p. 6882, dec. 2008.

SULliVAN, P.; PAQUETTE, K. J.; HOLT, N. L.; BLOOM, G. A. The relation of coaching context and coach education to coaching efficacy and perceived leadership behaviors in youth sport. Sport Psychologist, v. 26, n. 1, p. 122-134, 2012.

THOITS, P. A. Mechanisms linking social ties and support to physical and mental health. Journal of Health and Social Behavior, v. 52, n. 2, p. 145-161, 2011.

THON, R. A.; PASSOS, P. C. B.; COSTA, L. C. A. D.; PRATES, M. E. F.; NASCIMENTO JUNIOR, J. R. A. D.; VIEIRA, L. F. Leadership style in the context swimming coaches in the state of Paraná. Revista Brasileira de Cineantropometria e Desempenho Humano, v. 14, n. 5, p. 527-534, 2012. 
TOBAR, B. A. U. Analysis of preferred, perceived and observed leadership by coaches and athletes in soccer training: a case study. Cuadernos de Psicologia del Deporte, v. 15, n. 1, p. 197-210, 2015.

WEINBERG, R. S.; GOULD, D. Fundamentos da psicologia do esporte e do exercício. 6. ed. Porto Alegre: Artmed Editora, 2016.

Recebido em: 20/04/2017

Revisado em: 17/09/2017

Aprovado em: 18/09/2017

Endereço para correspondência:

marcelocardoso.esef@gmail.com

Marcelo Silva Cardoso

Universidade Federal do Rio Grande do Sul, Escola Superior de Educação Física.

Rua Felizardo, 750

Jardim Botânico

90690200 - Porto Alegre, RS - Brasil 